\title{
Tuberculosis, deprivation, and ethnicity in Leeds, UK, 1982-1997
}

\author{
R Parslow, N A El-Shimy, D B Cundall, P A McKinney
}

\begin{abstract}
Aims-To determine whether tuberculosis is increasing in frequency and to explore the association between deprivation, ethnicity, and tuberculosis in the city of Leeds.

Methods-Descriptive epidemiology and ecological analysis of a register of children and young people (0-18 years) diagnosed with tuberculosis from 1982 to 1997 in Leeds Health Authority.

Results-A total of 107 children were identified, 61 through contact tracing, to give an age and sex standardised incidence rate of 3.9 per 100000 per year. Rates decreased over the 16 year study period by an estimated $6.6 \%$ per year. The disease was more common in girls (56\%) and most frequent in 5-9 year olds, with respiratory disease accounting for the largest proportion (82\%). Children of south Asian origin (35\%) had a crude incidence rate of 25.7 per 100000 per year. The female:male ratios differed notably between south Asian (1.9:1) and nonsouth Asian children (1.02:1). For all subjects, univariate analyses showed significant positive associations between incidence and deprivation, population density, and ethnicity. There were no significant associations between deprivation, population density, and ethnicity and incidence of tuberculosis in south Asian children. For non-south Asian, mainly white children, only deprivation was significant. The proportion of non-south Asian children in the population was the overriding factor influencing incidence of tuberculosis.
\end{abstract}

Conclusions-Tuberculosis remains an uncommon disease in Leeds children. An unexpected finding was a relatively higher incidence in Asian girls compared to boys. Overall, ethnicity explains a high proportion of disease independently of deprivation and population density but for nonsouth Asian Leeds children the strongest risk factor is deprivation. (Arch Dis Child 2001;84:109-113)

Keywords: tuberculosis; ethnicity; deprivation

Epidemiology Group, Institute of

Epidemiology,

University of Leeds, 32

Hyde Terrace, Leeds

LS2 9LN, UK

R Parslow

P A McKinney

Correspondence to:

Dr Cundall

cundal@globalnet.co.uk

Accepted 21 August 2000 England and Wales showed a 35\% decline over five years. ${ }^{3}$ On the assumption that this trend would continue, the Joint Committee on to be a disappearing disease. ${ }^{1}$ In England and Wales the notification rate of tuberculosis has declined steadily from 1913 to $1987 .^{2}$ The 1983 survey of tuberculosis notification
Vaccination and Immunisation recommended stopping the school BCG programme by 1990 provided that the next survey showed a continuing downward trend. ${ }^{4}$ Unfortunately the national figures have shown no further decrease in notification rates. ${ }^{5}$

Tuberculosis has always been associated with poor living conditions and there is a recognised association between tuberculosis and poverty. ${ }^{67}$ Consistent patterns have emerged in national ${ }^{358}$ studies of tuberculosis showing higher rates in those from minority ethnic populations. ${ }^{9}$ For example, rates of tuberculosis of children of Bangladeshi origin are 25 times higher than those in the white population. $^{5}$

The 1991 census showed Leeds to be the second largest metropolitan district in the UK, covering an area of 217 square miles with a total population of 720000 . The area is divided into 33 electoral wards; the 12 most deprived wards constitute an inner city area with a total population of 235000 . This densely populated part of the city contains $60 \%$ of those claiming supplementary benefit, $60 \%$ of the unemployed, $75 \%$ of the minority ethnic populations, $50 \%$ of single parent families, but only $28 \%$ of the population. ${ }^{10}$

The city has the benefit of a central chest clinic with carefully maintained records of all cases of tuberculosis. An earlier retrospective study ${ }^{6}$ had used relatively crude measures of poverty (residence within a defined "urban priority area"). The present study used more appropriate epidemiological methods over a 16 year period.

\section{Methods}

Cases were ascertained using the tuberculosis notification register at Leeds Chest Clinic, clinical records for those treated at the chest clinic, and cross checked with the health visitors' register held at the clinic. Children included in the study were aged $0-18$ years, and diagnosed by a specialist physician at the chest clinic as having tuberculosis; their names were included on the tuberculosis register for the study period 1982-1997. Clinical assessment, tuberculin sensitivity, sputum microscopy and culture (when possible), and radiological assessment were used and the diagnosis made on the basis of all available information. Children were excluded if they were receiving antituberculous chemoprophylaxis, were infected with atypical mycobacteria, or when the diagnosis was changed by a chest physician on review. A standard proforma was used to abstract data from hospital case notes including full name, full address, and demographic details. Postcodes were validated using Quick- 


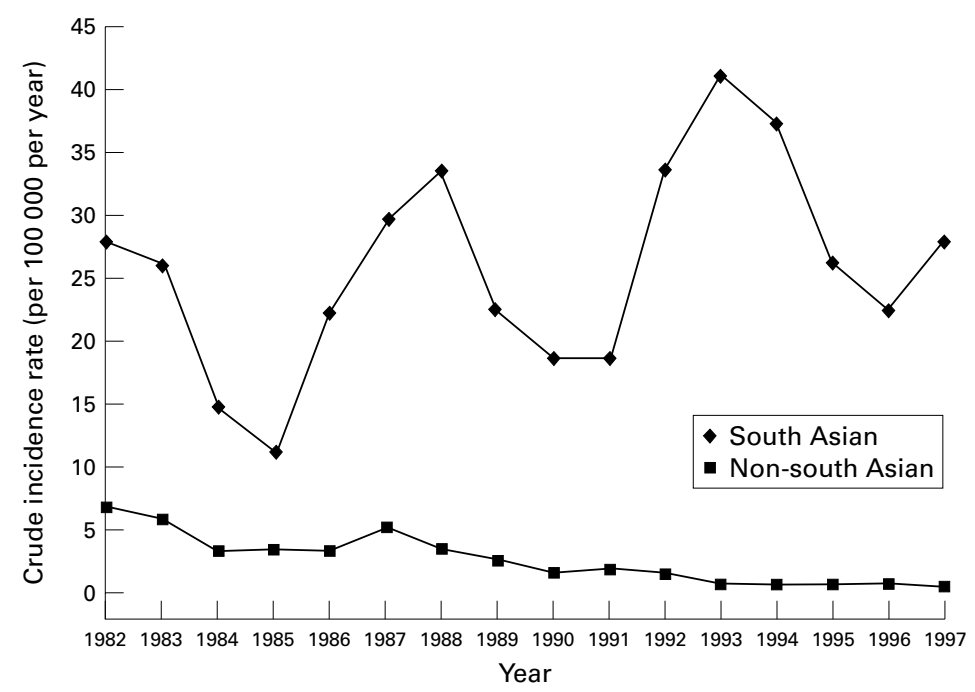

Figure 1 Incidence of tuberculosis in Leeds District Health Authority 1982-1997; three year moving average for south Asian and non-south Asian 0-18 year olds.

Address ${ }^{11}$; they were assigned to a 1991 census electoral ward via the Central Postcode Directory using the PC2ED facility at Manchester Information and Associated Services.

Each individual child was initially classified as being south Asian or not, using the Asian name analysis software Nam Pehchan. ${ }^{12}$ This was cross checked, where possible, with specialist tuberculosis health visitors who knew the children concerned.

Age sex standardised rates were calculated by ward for all cases of tuberculosis in $0-18$ year olds using the 1991 census (The 1991 census, ESRC purchase, Crown Copyright) to provide population denominators. For south Asians, a crude rate was calculated as denominator populations were not available by suitable age-sex strata. Three year moving averages of crude incidence were calculated for south Asian and non-south Asian populations for comparison. The average annual incidence rate was calculated for non-south Asians by regressing the log rate on year and expressing the exponential of the resulting coefficient -1 as a percentage.

For each ward in Leeds Health Authority, a deprivation score (the Carstairs Index ${ }^{13}$ ) and "ethnicity" (the proportion of non-white children under 19 years) were calculated using data from the 1991 census. Population weighted population densities were calculated by ward as this measure was considered to reflect more accurately the density at which the average person lives compared to area based population densities. ${ }^{14}$

Negative binomial regression modelling examined the association between ethnicity, deprivation, and population density on incidence of tuberculosis in the south Asian and nonAsian populations (Poisson models showed an unacceptable degree of over dispersion, especially when only considering south Asians whose numbers were very low). Each of these variables were divided into five groups having approximately equal populations; they were analysed in relation to all $0-18$ year olds (for which the age-sex stratified denominator
Table 1 Frequency, proportion, and incidence of children diagnosed with tuberculosis by age group and by sex

\begin{tabular}{lllrl}
\hline Age group $(y)$ & Males & Females & Total & (Col \%) \\
\hline $0-4$ & 11 & 15 & 26 & $(24)$ \\
$5-9$ & 16 & 23 & 39 & $(36)$ \\
$10-14$ & 5 & 9 & 14 & $(13)$ \\
15 & 4 & 3 & 7 & $(7)$ \\
$16-17$ & 6 & 6 & 12 & $(11)$ \\
18 & 5 & 4 & 9 & $(8)$ \\
Total (row \%) & $47(43.9)$ & $60(56.1)$ & 107 & \\
\hline
\end{tabular}

population data were available), south Asians (for whom only denominator population totals were available), and non-south Asians (also using unstratified population data for ease of comparison). These groups are based on values calculated for each of the 33 wards, hence the discontinuous range values. For all 0-18 year olds, deprivation and population density were each modelled with ethnicity (the proportion of non-white individuals in the population).

\section{Results}

DESCRIPTIVE EPIDEMIOLOGY

In the entire study cohort $(n=107), 42$ $(39.3 \%)$ were index cases while $61(57 \%)$ were found by contact tracing. Three cases were diagnosed following screening of new immigrants and one case was found during the school Heaf testing programme prior to BCG immunisation.

Table 1 describes the characteristics of the patient population by age and sex, showing the majority of children in the age group 5-9 years. Thirty eight $(35.5 \%)$ children in the study were of south Asian subcontinent origin, of whom 25 were girls and 13 were boys, giving a female to male ratio of 1.9:1. The female to male ratio in white children was 1.02:1.

Respiratory tuberculosis accounted for 88 (82\%) of the cases (57 non-south Asian, 31 south Asian) while 17 (16\%) had nonrespiratory tuberculosis (11 non-south Asian, six south Asian), predominately tuberculous lymphadenitis (11 cases; $65 \%$ of nonrespiratory tuberculosis).

Overall age-sex standardised incidence rate over the period 1982-1997 was 3.9 per 100000 per year $(95 \%$ confidence intervals 3.2 to 4.7 per 100000 per year). Crude incidence rates for $0-18$ year-olds of south Asian origin were 25.7 per 100000 per year (95\% confidence intervals 17.4 to 33.9 per 100000 per year), and 2.7 per 100000 per year $(95 \%$ confidence intervals 2.1 to 3.3 per 100000 per year) for the non-south Asian population. Figure 1 shows three year moving averages for these crude rates by year. There is a significant $13.3 \%$ average annual decrease in incidence for the non-south Asian population determined by linear regression (coefficient of $\log$ rate $=-0.142, \mathrm{p}=0.002)$. The overall average annual percentage decrease was $6.5 \%$ but was not significant (coefficient of log rate $=$ $-0.0678, \mathrm{p}=0.067)$.

ECOLOGICAL ANALYSIS

For the entire study population univariate analysis of each demographic factor by negative binomial regression modelling showed sig- 
Table 2 Univariate analysis of association between deprivation, population density, and ethnicity, and incidence of tuberculosis in 0-18 year olds in Leeds Health Authority, 1982-1997

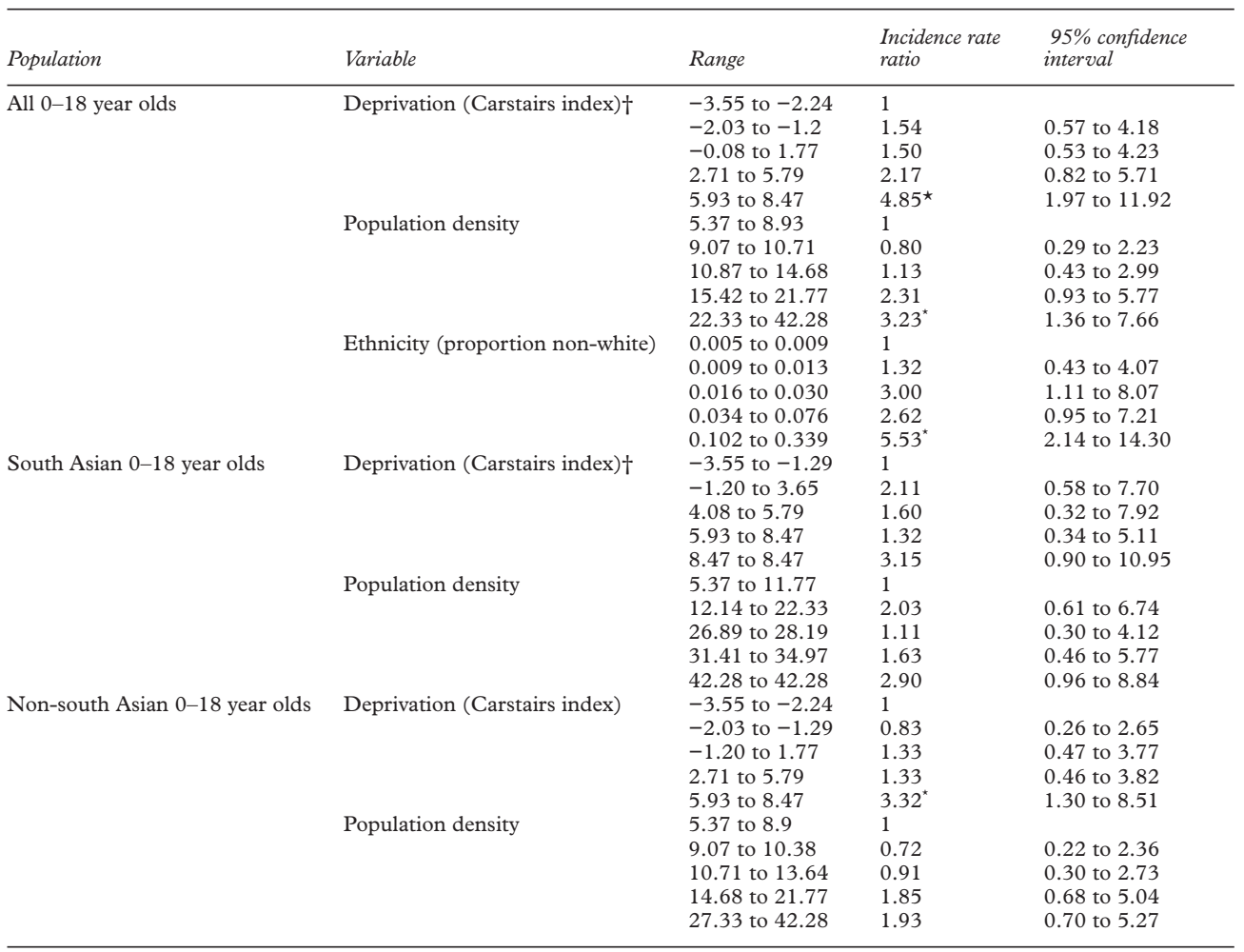

†Lower value, least deprived; higher value, most deprived

$\star$ Significant at $\mathrm{p}<0.05$.

nificant positive associations between incidence and ethnicity, deprivation, and population density (see table 2 ). When analysed separately there were no significant associations for south Asians; for non-south Asians a significant positive association was seen with deprivation but not population density.

In order to investigate further the relation between ethnicity and deprivation and ethnicity and population density, multivariate modelling was performed. Table 3 shows that ethnicity is independently and significantly associated with incidence of tuberculosis when modelled

Table 3 Ecological regression analysis of deprivation and population density in relation to ethnicity and incidence of tuberculosis in 0-18 year olds in Leeds District Health Authority, 1982-1997

\begin{tabular}{|c|c|c|c|}
\hline Variable & Range & $\begin{array}{l}\text { Incidence rate } \\
\text { ratio }\end{array}$ & $\begin{array}{l}95 \% \text { confidence } \\
\text { interval }\end{array}$ \\
\hline \multirow[t]{5}{*}{ Deprivation (Carstairs index) $\dagger$} & -3.55 to -2.24 & 1 & \\
\hline & -2.03 to -1.2 & 1.25 & 0.46 to 3.76 \\
\hline & -0.08 to 1.77 & 1.15 & 0.41 to 3.23 \\
\hline & 2.71 to 5.79 & 1.53 & 0.58 to 4.04 \\
\hline & 5.93 to 8.47 & 2.51 & 0.94 to 6.67 \\
\hline \multirow[t]{5}{*}{ Ethnicity (proportion non-white) } & 0.005 to 0.009 & 1 & \\
\hline & 0.009 to 0.013 & 1.23 & 0.76 to 3.76 \\
\hline & 0.016 to 0.030 & 2.16 & 0.76 to 6.18 \\
\hline & 0.034 to 0.076 & 2.02 & 0.71 to 5.72 \\
\hline & 0.102 to 0.339 & $3.12^{\star}$ & 1.12 to 8.61 \\
\hline \multirow[t]{5}{*}{ Population density } & 5.37 to 8.93 & 1 & \\
\hline & 9.07 to 10.71 & 0.60 & 0.23 to 1.60 \\
\hline & 10.8 to 14.68 & 0.40 & 0.13 to 1.25 \\
\hline & 15.42 to 21.77 & 1.22 & 0.46 to 3.27 \\
\hline & 22.33 to 42.28 & 0.73 & 0.23 to 2.37 \\
\hline \multirow[t]{5}{*}{ Proportion non-white } & 0.005 to 0.009 & 1 & \\
\hline & 0.009 to 0.013 & 1.27 & 0.43 to 3.77 \\
\hline & 0.016 to 0.030 & $3.15^{\star}$ & 1.01 to 9.85 \\
\hline & 0.034 to 0.076 & 2.18 & 0.75 to 6.39 \\
\hline & 0.102 to 0.339 & $6.72^{\star}$ & 1.88 to 24.04 \\
\hline
\end{tabular}

†Lower value, least deprived; higher value, most deprived. $\star$ Significant at $\mathrm{p}<0.05$. either with deprivation or population density, suggesting that the link with ethnicity is not explained either by deprivation or population density.

\section{Discussion}

Childhood tuberculosis is often regarded as unimportant in the epidemiology of tuberculosis, as more than $95 \%$ of children with tuberculosis are smear negative ${ }^{15}$ and therefore do not contribute to the immediate spread of the disease. However, childhood tuberculosis is necessarily a result of recent infection and therefore the presence of tuberculosis in children, particularly the younger age groups, reflects continuing transmission in the community. Infected children represent a pool from which a larger proportion of future cases of adult tuberculosis will arise. ${ }^{15}$ The World Health Organisation estimated that there were 7.96 million new cases and 1.87 million deaths in all age groups as a result of tuberculosis in $1997 .^{16}$

Under notification of tuberculosis in Britain is well recognised, but its extent is unknown. ${ }^{17}$ Leeds Health Authority has excellent cross checking systems to ensure accurate notification. The Consultant in Communicable Disease Control receives notifications of all positive cultures from the microbiology laboratories, histopathology laboratories, and the chest clinic. Local pharmacists inform the Consultant of all patients being prescribed combinations of antituberculous drugs. These mechanisms ensure continuous updating of the central tuberculosis register kept at the chest 
clinic and repeated local surveys have not detected any cases which were not already known to the chest clinic. It is therefore very unlikely that under notification is a significant problem in Leeds.

Our data showed that despite the fact that the total (adult and child) annual number of notified cases has been increasing in Leeds in the last three years of the study (J Watson, personal communication), the annual notifications for children alone have not shown a concomitant rise. The recent increase in adult notifications is likely to be caused by a combination of factors, for example an increase in homelessness, continued immigration, and increased reinfection of people visiting the Indian subcontinent.

Respiratory tuberculosis (82.2\%) was much more common than non-respiratory disease $(15.9 \%)$. There was an unexpected finding of a relatively higher number of south Asian girls with tuberculosis (male to female ratio 1.9:1 in south Asian and 1.02:1 in white children). Gender refers not only to physiological differences between the sexes, but also to the wide variety of behaviour, expectations, and roles attributed by culture to males and females. While much has been written about the influence of socioeconomic and cultural factors related to tuberculosis incidence, such studies have rarely analysed data by sex. ${ }^{18}$ There are some biological factors which may predispose females to tuberculosis infection. Animal studies suggest that the balance between the endogenous adrenal steroids cortisol and dehydroepiandrostenedione influences the balance of Th1 to Th2 cytokines which is thought to affect the immune response to tuberculosis. ${ }^{19}$ This would not explain the differential findings between south Asian and white girls in our study. Another recent study ${ }^{20}$ suggests that 25-hydroxycholecalciferol deficiency may contribute to the high occurrence of tuberculosis in Asians in west London. It is interesting to speculate about whether cultural factors, such as a tendency of Asian girls to spend more time at home, differentially affect vitamin D status, exposure to tuberculosis, or both. Prospective studies may help to explain the gender differential.

This study showed that the most affected age groups were children aged $0-4$ years $(24.3 \%)$ and 5-9 years $(39 \%)$. These figures are of particular significance as children have been the target of a number of primary preventive measures against tuberculosis infection, including selective BCG immunisation of infants at higher risk. The neonatal BCG programme has been reported to provide between approximately $64 \%{ }^{21}$ and at least $75 \%{ }^{22}$ protection against childhood tuberculosis, with an overall rate of protection estimated at $65 \%$ against respiratory disease but almost complete protection against disseminated disease. ${ }^{23}$ Although the BCG status of our cohort was not fully documented, a previous study from Leeds ${ }^{6}$ found that none of the active cases received neonatal BCG.

The finding from the ecological analysis that the overall risk of tuberculosis is linked to dep- rivation, population density, and ethnicity is not new. It is of interest, however, that the risk for ethnicity in the multivariate analysis was independent of both population density and deprivation and therefore not explained by these factors.

The study population was considered to be as complete as possible, but the sources of cases were not independent so the ascertainment could not be formally tested. The numbers included were relatively small and caution in interpretation of the results is therefore important; however, the publication of similar findings from an independent study of tuberculosis ${ }^{9}$ lends weight to our results.

For the descriptive epidemiology, individuals were flagged as south Asian based on their name, a procedure which may be open to misclassification. However, the Nam Pehchan program,$^{24}$ which identifies part name (stem) or complete matches from a data dictionary of Asian names is used by the City of Bradford Metropolitan Council and Bradford Health Authorities in West Yorkshire and is particularly appropriate for the population in this study. Further checks were made with health care professionals dealing with the Asian families, along with visual inspection of the names; we therefore consider it unlikely that any major misclassification will have occurred.

The ecological analyses used census based variables to measure ethnicity, that is, the proportion of non-white children in the population; it should be noted that measuring summary characteristics of a population residing in an area, however restricted in size, does not necessarily represent the condition of any single individual. ${ }^{25}$ Despite this, a number of area based studies in the UK, ${ }^{26}{ }^{27}$ although not all, ${ }^{28}{ }^{29}$ have shown that census based investigations are a reasonable method of investigating the risk of disease in relation to population characteristics.

Examining our childhood populations of south Asians and non-south Asians (mainly white) separately, we found no links between deprivation or population density for south Asian children, reflecting a similar pattern shown in the combined childhood and adult population in Birmingham. ${ }^{9}$ For non-south Asian children there was an association with deprivation but not population density. These findings from the ecological analysis, in conjunction with the differential time trends, suggest that disease aetiology and the risk profile may be different for south Asian compared to other children. Our observations make it important for future analyses of overall trends and incidence rates to take account of ethnicity. Failure to do so will lead to misunderstanding of the dynamics of tuberculosis infection in the population.

Thanks to Dr John Watson and staff at the Leeds Chest Clinic for access to the data and to Dr Martin Schweiger, Consultant in Communicable Disease Control, Leeds Health Authority for advice.

1 Silverman M. Childhood tuberculosis in Britain. BMf 1988;297:1147-8.

2 Meredith S. National survey of notification of tuberculosis in England and Wales in 1988. Thorax 1993;48:304. 
3 Medical Research Council, Tuberculosis and Chest Disease Unit. Tuberculosis in children in a national survey of notification in England and Wales in 1983. Arch Dis Child 1988;63:266-76.

4 Department of Health and Social Security. The school BCG vaccination programme (letter to Health Authorities, DA(85) 27 and annex). London: DHSS, 1985.

5 Medical Research Council, Cardiothoracic Epidemiology Group. Tuberculosis in children: a national survey of notifroup. Tuberculosis in children: a national survey of notification in Englan

6 Cundall DB, Pearson SB. Inner city tuberculosis and immunisation policy. Arch Dis Child 1988;63:964-6.

7 Spence DPS, Hotchkiss J, Williams CSD, Davies PDO. Tuberculosis and poverty. BMF 1993;307:759-61

8 Nunn J, Darbyshire JH, Fox W, et al. Changes in annual tuberculosis notification rates between 1978/9 and 1983 for the population of Indian subcontinent ethnic origin resident in England. $f$ Epidemiol Community Health 1986;40:357-63.

9 Hawker JI, Bakhshi SS, Shaukat A, Farrington P. Ecological analysis of ethnic differences in relation between tuberculosis and poverty. BMF 1999;319:1031-4

10 Goldman JM, Teale C, Cundall DB, et al. Childhood tuberculosis in Leeds, 1982-1990: social and ethnic factors and the role of contact clinics in diagnosis. Thorax 1994;49. 184-5.

11 QAS Systems Ltd, 12-14 Brindley Road, Manchester M16 9HQ.

12 NAM PEHCHAN: South Asian Names (Indian Subcontinent). A computer program to identify and analyse south Asian names, version 1.1. City of Bradford Metropolitan District Council Computer Services Division, 1996.

13 Carstairs V, Morris R. Deprivation and health in Scotland. Aberdeen: Aberdeen University Press, 1991.

14 Dorling D, Atkins D. Population density, change and concentration in Great Britain 1971, 1981 and 1991. Studies on medical and population subjects, no. 58. London: ies on medical

15 Rie AV, Beyers N, Gie RP, et al. Childhood tuberculosis in South Africa: burden and risk factors. Arch Dis Child 1999; 80:433-7.
16 Dye C, Scheele S, Dolin P, Pathania V, Raviglione MC. Consensus statement: global burden of tuberculosis: estimated incidence, prevalence and mortality by country. estimated incidence, prev

17 Watson JM. Tuberculosis in Britain today. BMF 1993;306: 221-2.

18 Hudelson P. Gender differentials in tuberculosis: the role of socio-economic factors. Tubercle and Lung Disease 1996;77: $391-400$.

19 Rook GA, Hernendez-Pando R. Pathogenetic role in human and murine tuberculosis of changes in the peripheral metabolism of glucocorticoids and anti-glucocorticoids. Psychoneuroendocrinology 1997;22(suppl):S109-13.

20 Wilkinson RJ, Llewelyn M, Toossi Z, et al. Influence of vitamin $\mathrm{D}$ deficiency and vitamin $\mathrm{D}$ receptor polymorphisms on tuberculosis among Gujarati Asians in west London: a case-control study. Lancet 2000;355:618-21.

21 Curtis HM, Leck I, Bamford FN. Incidence of childhood tuberculosis after neonatal BCG vaccination. Lancet 1984; 1:145-8.

22 Packe GE, Innes JA. Protective effect of BCG vaccination in infant Asians: a case-controlled study. Arch Dis Child 1988; 63:277-81.

23 Clarke A, Rudd P. Neonatal BCG immunisation. Arch Dis Child 1992;67:473-4.

24 Cummins C, Winter H, Cheng K-K, Maric R, Silcocks P, Varghese C. An assessment of the Nam Pehchan computer program for the identification of names of south Asian origin. F Public Health Med 1999;21:401-6.

25 Greenland S, Morgestern H. Ecological bias, confounding and effect modification. Int f Epidemiol 1989;18:269-74.

26 Spencer N, Bambang S, Logan S. Socioeconomic status and birthweight: comparison of an area-based measure with the Registrar General's social class. F Epidemiol Community Health 1999,53:495-8.

27 Haining $\mathrm{R}$, Wise $\mathrm{S}$, Blake, $\mathrm{M}$. Constructing regions for small area analysis: material deprivation and colorectal cancer. $\mathcal{F}$ Public Health Med 1994;16:429-38.

28 Saul C, Payne N. How does the prevalence of specific morbidities compare with measures of socio-economic status at bidities compare with measures of socio-economic status

29 Collins SE, Haining RP, Bowns IR, et al. Errors in postcode to enumeration district mapping and their effect on small area analyses of health data. I Public Health Med
1998;20:325-30.

\section{2nd World Congress of the Pediatric Thoracic Disciplines}

\section{April 26-8, 2000; Izmir, Turkey}

Further details: Prof Dr Oktay Mutaf, Ege University Faculty of Medicine, Pediatric Surgery Department. Fax: +90232 3751288; email: omutaf@med.rgr.rdu.tr 